Revue scientifique sur la conception et l'aménagement de l'espace

19 | 2018

L'imagerie du paysage

\title{
Des îles à la montagne : paysages publicitaires de la feta
}

From Islands to Mountains: The Advertising Landscapes of Feta

\section{Katerina Seraïdari}

\section{OpenEdition}

\section{Journals}

Édition électronique

URL : http://journals.openedition.org/paysage/361

DOI : $10.4000 /$ paysage.361

ISSN : 1969-6124

\section{Éditeur :}

École nationale supérieure du paysage de Versailles-Marseille, Institut national des sciences appliquées Centre Val de Loire - École de la nature et du paysage, École nationale supérieure d'architecture et de paysage de Bordeaux, École nationale supérieure d'architecture et de paysage de Lille, Agrocampus Angers

\section{Référence électronique}

Katerina Seraïdari, "Des îles à la montagne : paysages publicitaires de la feta », Projets de paysage [En ligne], 19 | 2018, mis en ligne le 01 décembre 2018, consulté le 28 novembre 2019. URL : http:// journals.openedition.org/paysage/361 ; DOI : 10.4000/paysage.361

Ce document a été généré automatiquement le 28 novembre 2019.

Projets de paysage 


\title{
Des îles à la montagne : paysages publicitaires de la feta
}

\author{
From Islands to Mountains: The Advertising Landscapes of Feta
}

\author{
Katerina Seraïdari
}

1 Afin de faciliter la construction d'une politique agricole commune, le règlement européen de 1992 a créé l'appellation d'origine protégée (AOP) et l'indication géographique protégée (IGP). Celles-ci instaurent une relation entre le produit et son espace de production, qui implique «la non-reproductibilité » et «la mise en place d'une zone délimitée, hors de laquelle la fabrication du produit considéré est juridiquement impossible» (Frayssignes, 2007, p. 148). Ces signes de qualité, qui introduisent un principe de protection géographique et qui correspondent à un cahier des charges précis, constituent une extension des lois sur la «propriété intellectuelle » : ils associent un terroir à une production qui lui est propre et qui est considérée comme inimitable et exclusive.

2 En 1994, l'État grec demande à la Communauté européenne le titre d'AOP pour la feta. Afin de vérifier que ce produit n'est pas générique, la commission procède à une enquête auprès de 12800 nationaux des douze pays qui font alors partie de la Communauté européenne : pour $37,2 \%$ des enquêtés il s'agit d'un nom générique, $35,2 \%$ considèrent que ce produit a une origine géographique particulière et le reste n'exprime pas d'avis sur le sujet. Compte tenu des résultats, l'AOP est attribuée en 1996. La feta doit dorénavant être fabriquée avec du lait produit en Grèce continentale, à une exception près : Lesbos, seule île reconnue comme productrice d'une feta AOP.

Devant les protestations du Danemark, de l'Allemagne et de la France qui produisent de la feta depuis les années 1960, la commission ouvre une seconde enquête en 1999. De nouveaux critères apparaissent, comme le niveau de production et de consommation dans les différents pays européens, ainsi que la nature des publicités concernant ce produit. Le comité scientifique établit même le seuil à partir duquel une indication géographique devient nom générique: il faut que seule une toute petite partie du public attribue une origine géographique à un produit pour que ce dernier soit catégorisé comme générique. Cette position explique, d'ailleurs, la première décision de 
la commission qui avait refusé de caractériser comme générique un produit auquel seul un tiers des personnes interrogées assignaient une origine géographique spécifique.

4 Cette nouvelle enquête a montré que la feta qui est produite au Danemark, en Allemagne et en France porte des noms à connotation grecque et que son marketing met en avant une imagerie typiquement grecque. Après avoir conclu que dans la perception des consommateurs le nom «feta » évoque encore une origine grecque, la commission a donc confirmé l'AOP en 2003 (O'Connor, 2004, p. 96-101). Un délai de cinq ans a été laissé aux autres producteurs européens de feta qui avaient le choix entre trouver un autre nom pour leurs produits ou en arrêter la fabrication.

5 Si l'image publicitaire d'un produit peut devenir un argument dans la bataille commerciale et juridique autour des AOP, cela montre bien que l'imagerie promotionnelle et par conséquent les paysages qui sont choisis, afin d'associer un produit à une ambiance ou/et à une origine, ne sont pas neutres. Bien au contraire, les paysages jouent un rôle essentiel dans la construction de l'identité géographique que les producteurs et les consommateurs assignent à un produit.

6 Depuis les années 1990, le désir de typicité, d'historicité et d'identification géographique ne fait que croître (Poulain, 2002). Le client ne s'intéresse pas seulement au prix ou au goût du produit, il veut aussi pouvoir le mettre en rapport avec un lieu et une histoire. Le plus souvent, c'est l'emballage qui raconte l'histoire de la famille qui a fondé l'entreprise, ou du lieu de production présenté sous une forme idyllique ou emblématique. L'évocation iconographique d'un lieu constitue, à la fois, une forme de labellisation et une preuve de transparence, dans la mesure où elle offre au produit un ancrage géographique, plus ou moins imaginaire selon les cas. Comme nous le verrons, le lieu de production ou le paysage qui figurent sur l'emballage localisent, circonscrivent et valorisent. Cette mise en scène des paysages correspond à une stratégie commerciale visant à rendre le produit plus attractif, mais aussi à une exigence d'inscription géographique de la part des consommateurs qui se méfient de l'opacité d'un marché de plus en plus global et industrialisé: plus le risque d'une provenance incertaine guette les produits, plus la valorisation de la typicité et de la « régionalité » devient importante.

\section{Méthodologie}

7 En m'appuyant sur un corpus d'une quarantaine de marques grecques mais aussi étrangères, mon but est d'examiner le marché européen de la feta après l'obtention de l'AOP. La perspective est donc synchronique. À l'exception de la marque Salakis, toutes les marques européennes mentionnées ici commercialisent une feta AOP fabriquée en Grèce. Par conséquent, les marques étrangères sont généralement des marques de commerce, alors que les marques grecques sont tantôt des marques de fabrique (la marque portant le nom du fabricant), tantôt des marques de commerce. En ce qui concerne la sélection des marques, je n'examine ni celles qui optent pour des emballages ne montrant que des morceaux de feta accompagnés d'autres ingrédients ni celles dont l'imagerie renvoie à la Grèce antique.

8 Les emballages neutres sont majoritaires sur le marché grec. Par exemple, la coopérative épirote Dodoni, qui est le plus grand producteur de fromages en Grèce, n'utilise que ce type d'emballage pour la feta qu'elle commercialise. De nombreuses raisons peuvent expliquer cette tendance. Tout d'abord, la feta s'achetait 
traditionnellement en Grèce à la coupe ; ce n'est que depuis quelques années que les Grecs l'achètent préemballée et en grande surface ${ }^{1}$. Encore aujourd'hui dans les supermarchés, on peut trouver de la feta en tonneau qui est vendue à la coupe (hyma en grec).

Photo 1. Feta à la coupe dans un supermarché athénien (Nea Kifissia)

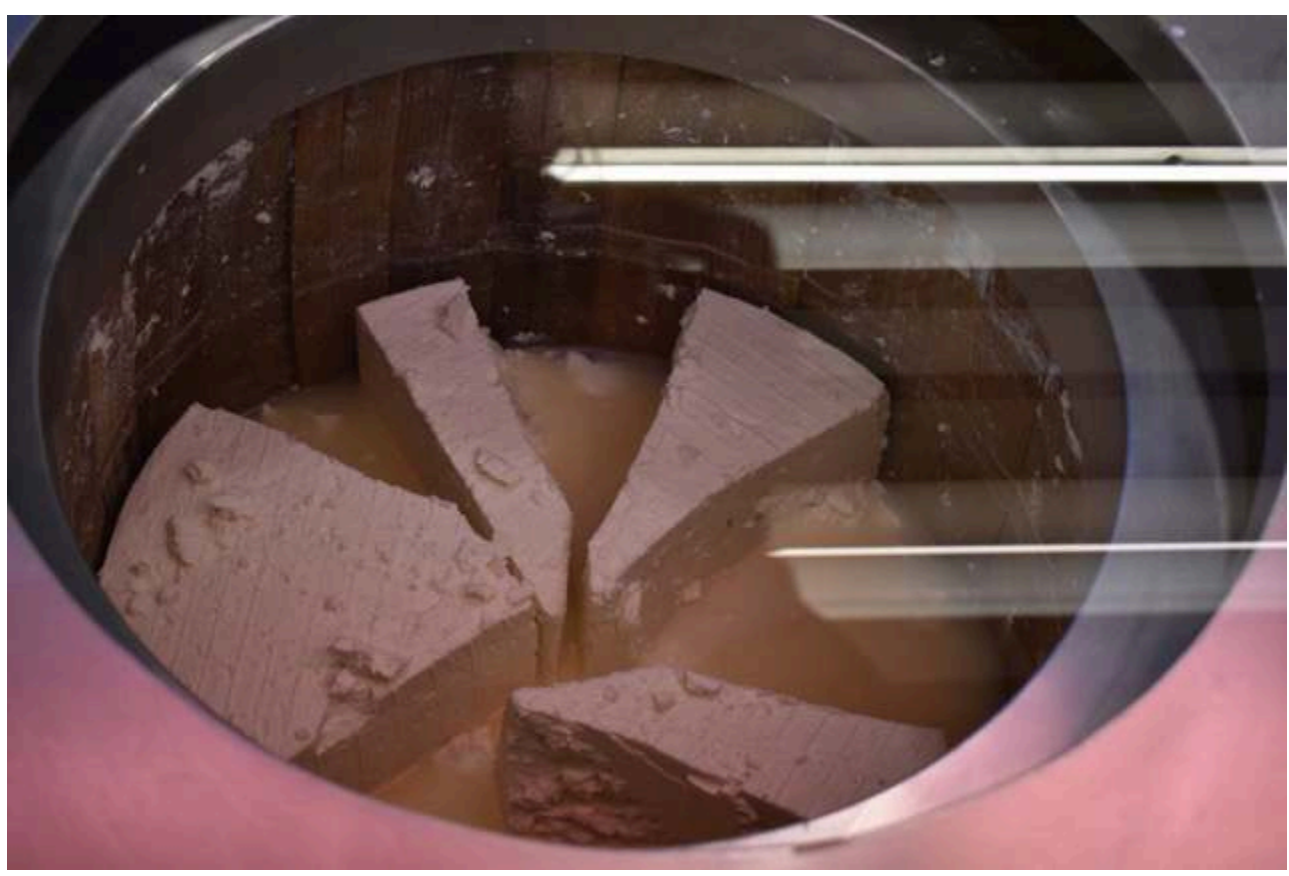

25 septembre 2018.

Photographie d'Alkisti Kyriakopoulou.

Deuxièmement, si pour les Européens la feta est simplement grecque, pour les Grecs ce fromage est doté de caractéristiques spécifiques selon la région de provenance : c'est plus cette dernière qui compte en tant que critère d'achat que la marque ou l'emballage. Même quand les Grecs achètent à la coupe dans un supermarché, ils ont le choix entre des feta qui proviennent de différentes régions.

Troisièmement le minimalisme peut aussi être interprété comme un signe de qualité : un bon produit n'a pas besoin de séduire. Les modes de distribution jouent également un rôle. Par exemple, en ce qui concerne le vin, une étiquette sobre suffit "pour le circuit hôtellerie-restaurant où un sommelier reste l'intermédiaire»; toutefois, on trouve les étiquettes de vin les plus minimalistes dans les hard discounters (Moser, 2011, p. 131). Un emballage neutre peut donc qualifier tant un produit « raffiné » que celui qui se veut « économique »; il est également associé au produit artisanal, qui est diffusé localement.

11 Mon article s'attache à comparer deux types de paysages, ceux qui sont représentés sur les emballages de la feta dans des pays européens comme la France, la Belgique et l'Allemagne, et ceux qui ont vocation à séduire le public grec. Je n'ai donc retenu que les marques dont le packaging évoque un paysage. Cependant, je ne me référerai pas à toutes, puisqu'elles reproduisent des schémas iconographiques récurrents.

12 Il faut également préciser que je me suis intéressée uniquement aux emballages, et non aux différents types de campagne publicitaire. Mon approche est donc différente de 
celle de Dobremez, Rapey, Candau et Ginelli (2013) qui, afin d'examiner les liens entre produit et paysage et d'identifier les "éléments matériels du paysage ", utilisent des photographies provenant de dépliants, d'ouvrages et de cartes postales, tout en s'appuyant sur des entretiens avec les éleveurs.

Les emballages que j'ai examinés reflètent des stratégies de communication et de marketing. Ils révèlent la manière dont le marché de la feta se structure au sein de l'Union européenne : dans ce cadre, le choix du paysage devient un enjeu économique et pose des questions qui vont au-delà des conditions de fabrication et qui touchent tant à l'industrie du tourisme qu'aux préoccupations religieuses. Ainsi, mon analyse a peu de choses en commun avec celle d'Yves Michelin (2008) qui a proposé une "démarche de construction collective d'une étiquette ", à partir d'éléments isolés et extraits d'un corpus de quatre cents étiquettes collectées auprès de vendeurs de fromages et de collectionneurs. Les personnes impliquées dans son enquête assemblent des "morceaux de paysage» en relation avec des fromages AOC d'Auvergne. Lydia Menadier (2010) s'intéresse également à la sensibilité paysagère des agriculteurs de la même filière AOC. Ces études, qui cherchent à définir le rapport des personnes à l'espace, privilégient les enquêtes de terrain et l'échelle locale. Les paysages qu'elles examinent acquièrent une fonction émancipatrice; d'ailleurs, elles ne citent aucune marque - comme s'il fallait prendre de la distance par rapport au cadre marchand. En revanche, la question que mon article pose est de savoir dans quelle mesure le paysage, une fois instrumentalisé par la publicité, permet de saisir un marché en pleine expansion, celui de la feta. Les marques et les images promotionnelles qu'elles mettent en avant sont omniprésentes dans mon texte. De ce point de vue, je suis plus dans le sillage de Roland Barthes (1964) qui analyse l'emballage des pâtes Panzani afin de mieux comprendre le positionnement d'un produit « italien » sur le marché français.

14 Malgré ces choix méthodologiques, mon approche se veut ethnographique : ce sont mes enquêtes de terrain sur les épiceries grecques et turques de Bruxelles (2009-2015) qui m'ont poussée à m'intéresser à l'offre des produits laitiers. Dès le début, je me suis aperçue que les marques de feta que ces commerces vendaient étaient inconnues en Grèce. Mes enquêtes de terrain, qui ne sont pas détaillées ici, constituent une étape en amont qui m'a permis de prendre conscience de la division du marché de la feta.

En effet, il faut faire la distinction entre une feta "domestique» et une feta « touristique » qui est consommée hors de la Grèce, commercialisée par des entreprises européennes, mais aussi grecques. Cependant, la feta ici appelée «touristique » est consommée non seulement par des acheteurs qui n'ont pas de liens particuliers avec la Grèce, mais aussi par les immigrés et les expatriés grecs. L'internationalisation de certains produits, jadis considérés comme "ethniques», est liée à l'immigration, puisque les immigrés ont organisé leur commerce dans le pays d'accueil afin de satisfaire en premier lieu leurs propres besoins (Seraïdari, 2012). Cette feta est donc autant « immigrée » que «touristique » mais, pour des raisons que j'expliquerai plus loin, seul le deuxième adjectif a été retenu.

La feta «touristique» est industrialisée. En revanche, le marché de la feta « domestique » inclut aussi des petits producteurs qui ont peu de moyens pour diffuser leurs produits. Ce marché est particulièrement difficile à saisir, à cause du grand nombre et de l'éparpillement des producteurs: aucun supermarché du pays ne peut avoir toutes les marques industrielles, locales et artisanales qui existent sur le marché grec. Il y a donc un décalage évident entre la réalité de l'offre et les points de vente, qui 
ne laissent paraître qu'une partie de cette offre : les produits industrialisés ont plus de visibilité et sont plus présents sur les rayons. Dans ce cas, j'ai choisi de ne pas effectuer d'enquêtes de terrain, considérant qu'Internet pourrait être un outil plus approprié pour se rendre compte de cette diversité. Ce choix méthodologique m'a amenée à découvrir des marques que beaucoup de Grecs ne connaissent pas. Ainsi, quand j'ai demandé à une étudiante de dix-huit ans, Alkisti Kyriakopoulou, de photographier des emballages de feta dans les supermarchés à proximité de son domicile athénien, elle n'a pu trouver que deux marques (Karalis et Minerva) sur la dizaine que je lui avais indiquée.

Les images qui accompagnent mon article sont donc de trois ordres: les photographies de Kyriakopoulou reflètent l'offre des supermarchés athéniens, mes photographies l'offre des épiceries turques de Bruxelles, alors que les images publicitaires, qui proviennent d'Internet, concernent des produits qui ne sont pas associés à un lieu de vente spécifique.

Les enjeux qui m'intéressent sont d'ordre visuel, mais j'accompagne leur analyse d'éléments relatifs aux entreprises qui commercialisent ces produits. Les discours de quelques fabricants sont ainsi mis en parallèle avec les images publicitaires qu'ils ont choisies pour leurs emballages. L'objectif de cet article est d'analyser comment chaque marque se positionne dans le marché de la feta, mais aussi comment ce positionnement est également visuel et " paysager ».

\section{Une marque allemande : Sirtakis}

Les immigrés grecs ont ouvert les premières épiceries à Bruxelles dans les années 1960, alors que l'entreprenariat turc ne s'est développé qu'à partir des années 1980. Dans un premier temps, ces magasins se trouvaient dans les quartiers ouvriers où il y avait une concentration de populations grecque et turque, et leur offre était « ethnique ». Mais depuis les années 2000, des épiceries tenues par des commerçants turcs ont fait leur apparition dans les quartiers bruxellois aisés. Ces boutiques, ainsi que les quelques épiceries tenues par des Grecs qui survivent encore, ont largement perdu leur caractère ethnique et sont actuellement fréquentées par une clientèle très variée (Seraïdari, 2012).

20 Les épiceries tenues par des Turcs proposent souvent des produits laitiers de la marque Gazi. Ce qui les caractérise est une iconographie orientalisante (mosquée, minaret, femmes en costume traditionnel) et des logos où la couleur dominante est le rouge couleur du drapeau turc. 
Photo 2. Fromage Gazi, avec mosquée

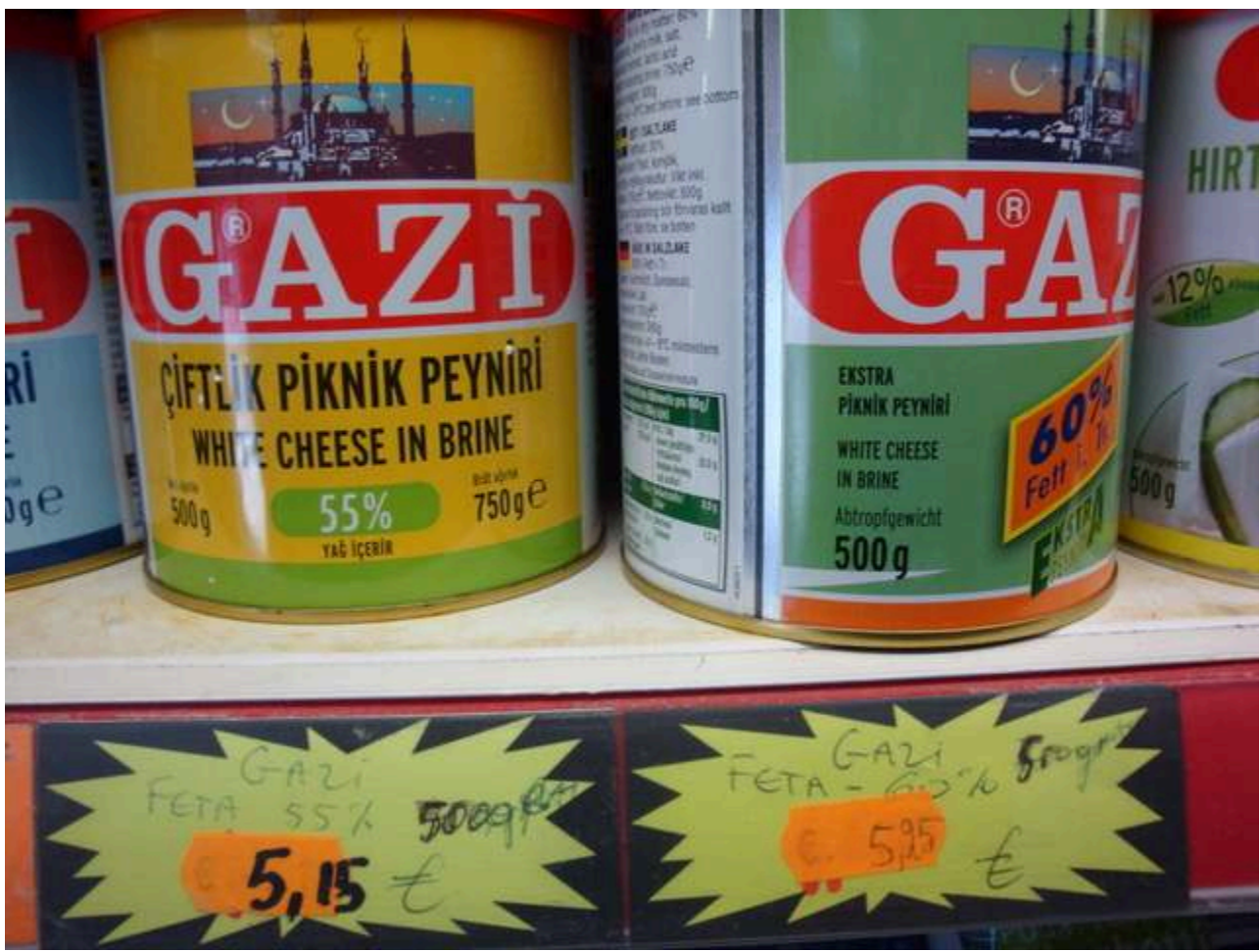

Les informations sur la boîte étant en turc et en anglais, l'épicier turc de cette épicerie bruxelloise (quartier d'Ixelles) a ajouté sur l'étiquette du prix qu'il s'agit de feta, 3 mars 2013.

Photographie de Katerina Seraïdari.

Après des recherches sur Internet, j'ai découvert qu'Eduardo Garcia, un entrepreneur de père espagnol et de mère allemande, a fondé en 1974 l'entreprise allemande Garmo qui commercialise, entre autres, deux gammes de produits : Gazi et Sirtakis. La marque Sirtakis, qui est inspirée de la tradition culinaire grecque, ne propose pas seulement de la feta, mais aussi du haloumi (fromage chypriote), du tzatziki et des yaourts.

La feta Sirtakis est conditionnée à Stuttgart dans une entreprise fondée par un entrepreneur, Eduardo Garcia, dont les origines ne sont ni grecques ni turques, mais qui est considéré comme étant un «juif de Turquie » par la majorité des épiciers turcs de Bruxelles que j'ai interrogés. Selon Stéphane de Tapia (2005, p. 63), ce malentendu est à l'origine du parcours professionnel d'Eduardo Garcia: en 1969, Garcia a sympathisé avec des grossistes turcs d'Istanbul qui pensaient qu'il était juif, à cause de son nom espagnol.

23 La production de la feta en Allemagne commence en 1972 (O'Connor, 2004, p. 98, note 13). Tout laisse à penser que Sirtakis est une des premières marques de feta produites en Allemagne. Toutefois, l'Allemagne, à l'instar des autres pays européens qui produisaient ce fromage avant l'assignation de l'AOP, a dû définitivement arrêter la production de la feta en 2010. La marque Sirtakis commercialise aujourd'hui une feta AOP produite en Grèce. Sur un site Internet ${ }^{2}$, les techniciens grecs sont présentés comme les garants, d'une part, de la haute qualité des produits Sirtakis et, d'autre part, de la sauvegarde des vieilles recettes de l'île de Rhodes qui servent à la fabrication de ce fromage. Ce discours lie technologie moderne et tradition - même si l'île de Rhodes ne fait pas partie des territoires grecs où une feta AOP peut être fabriquée. Nous avons ici une indication vague du lieu de provenance de la recette suivie, mais rien sur le lieu de 
fabrication. En effet, la plupart des marques de commerce européennes ne précisent pas le lieu de fabrication en Grèce, comme si la question du terroir n'était pas importante.

Le nom de cette marque évoque une danse grecque, le sirtaki, qui est devenue célèbre en Europe occidentale dans les années 1960 : le sirtaki a contribué à populariser la cuisine et les restaurants grecs qui faisaient danser les clients au son de cette musique caractéristique (Seraïdari, 2012). D'ailleurs, il existe en France une gamme de biscuits fabriqués avec des raisins de Corinthe, appelée Sirtaki, et que des marques, comme Lu et Saint-Michel, commercialisent.

L'emballage de cette feta se divise en deux moitiés. Sur la partie supérieure, on voit la mer, le ciel, deux silhouettes d'îles au fond, puis le $\operatorname{logo}^{3}$ placé au centre, ainsi que l'origine de ce fromage («original griechischer »); cette partie, qui est épurée, est dominée par des nuances de bleu et de blanc. En revanche, la partie inférieure est plus chargée iconographiquement. Sur une table en bois qui est partiellement couverte d'une nappe à carreaux bleus et blancs, repose une assiette dans laquelle on trouve un gros morceau et trois petits cubes de feta avec quelques feuilles de basilic. Juste audessus d'un cube de feta, à droite, apparaît un drapeau grec stylisé dans un cercle avec, à sa base, une banderole qui décrit le goût du produit («mild aromatisch»). Quant au sigle AOP, il est placé à gauche, en bas de l'image.

Photo 3. Publicité sur Internet pour la feta Sirtakis

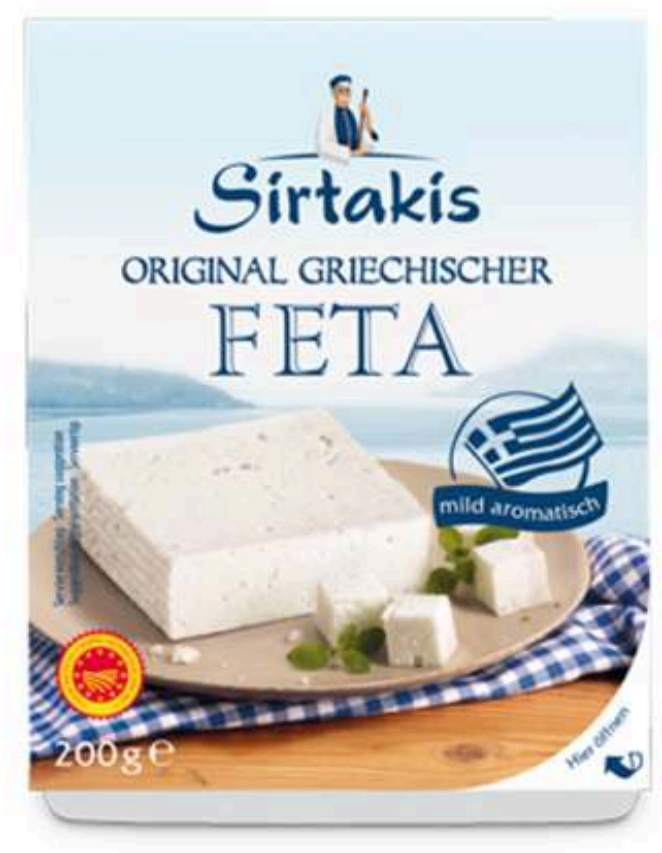

La plupart des marques de feta qui circulent en Europe ont recours à cette iconographie qui est devenue typique : mer (souvent avec des reflets de soleil), ciel bleu, phares, moulins à vent ou églises blanches à dômes bleus. Très souvent, comme dans le cas de Sirtakis, la table où le plat est posé devient elle-même un balcon qui permet d'admirer 
la vue d'en haut: le consommateur est donc mis dans une position surplombante, comme si le paysage se déployait à ses pieds.

Les illustrations sur les emballages de la feta, choisies par les marques étrangères, sont les mêmes que celles que l'on retrouve sur les yaourts à la grecque fabriqués en Europe depuis les années 1990. Elles s'inspirent de l'architecture des Cyclades et plus particulièrement de Santorin. Le paysage se donne à voir sous une forme de « représentation synecdochique ( (Bigando, 2006) : une partie choisie pour représenter le tout. Ce processus de simplification de la réalité paysagère, qui obéit à un souci de lisibilité auprès des consommateurs, fait de Santorin un élément référentiel indispensable - cette île signifie l'ensemble du territoire grec. Toutefois, les paysages cycladiques marquent les emballages non seulement de la feta mais aussi d'autres produits laitiers qui renvoient à la Grèce, même quand il n'y a pas d'AOP.

\section{Cyclades et feta « touristique »}

L'emballage est un support qui facilite le conditionnement, la conservation et le transport. Il sert souvent à faire se démarquer le produit ou à souligner sa singularité ; mais son rôle consiste également à lui permettre d'entrer dans une catégorie définie par un ensemble d'images familières. Des marques comme Salakis-Lactalis (France) choisissent également pour leur packaging cette imagerie qui est devenue typique : le mot «feta » n'y figure pas, conformément à la réglementation de l'Union européenne, puisque ce produit n'est pas AOP. Facilement reconnaissable, l'iconographie cycladique permet alors à un fromage fabriqué en France (et présenté comme " $100 \%$ brebis») d'acquérir un parfum d'authenticité grec.

Le paysage cycladique vise à rendre identifiable (en tant que grec) un produit qui n'est pas toujours produit en Grèce : c'est le cas pour le fromage Salakis, mais aussi pour les yaourts à la grecque ou les biscuits Sirtaki dont l'emballage représente une église cycladique. Le paysage représenté devient ainsi «trompeur ", dans la mesure où son potentiel référentiel est utilisé pour induire le consommateur en erreur. Cependant, la loi interdit la mention du mot "feta" sur l'emballage mais pas l'utilisation d'une imagerie grecque. Ce qui est protégé est le produit agricole (le contenu) et non l'image qui figure sur le packaging (le contenant).

30 Ces représentations paysagères se sont retrouvées au centre d'un débat en août et septembre 2017, quand la presse belge, allemande et française a commenté la disparition des croix surmontant les églises cycladiques sur les emballages des yaourts à la grecque ; cette retouche aurait été faite par respect pour la diversité religieuse. Les réseaux sociaux et les médias l'ont parfois assimilée à une censure révélant une « christianophobie » croissante. 


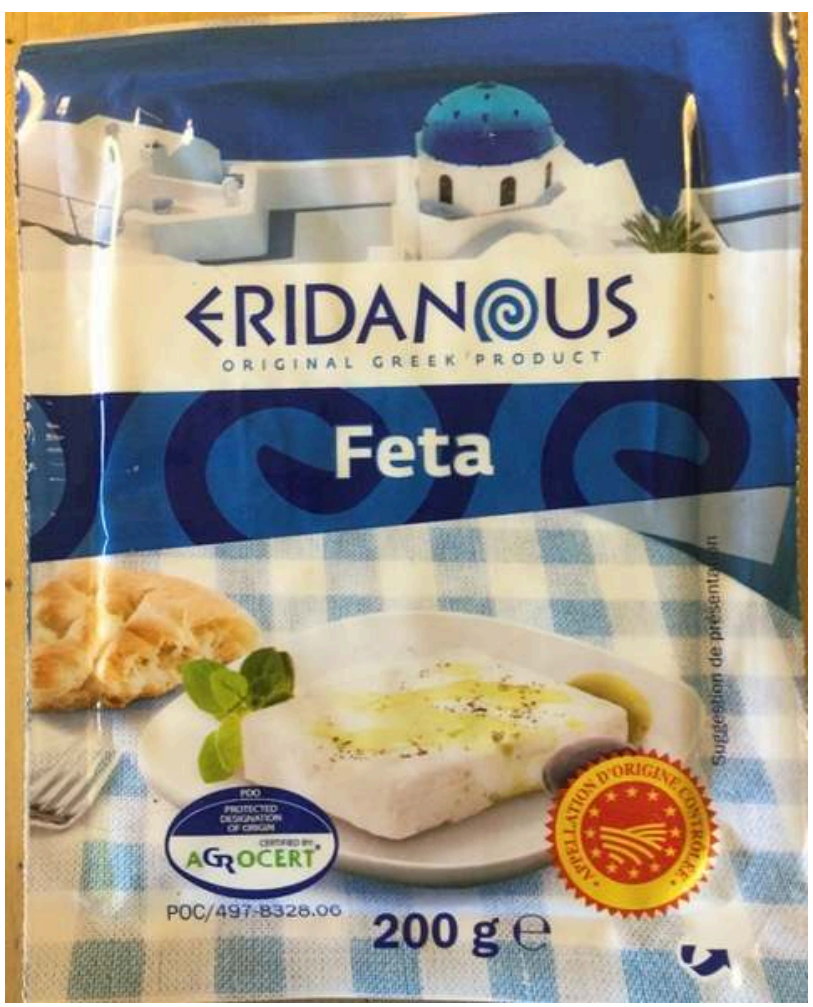

La table est devenue un balcon qui surplombe le paysage cycladique alors que l'église est représentée sans croix. La marque Eridanous est commercialisée par Lidl. Si l'enseigne alimentaire allemande distribue Eridanous dans différents pays européens, en Grèce, elle commercialise une autre marque, la feta Galpo, dont l'emballage reproduit des scènes pastorales.

Dans un article du journal La Croix, une autre affaire est mentionnée, celle d'Aldi qui a dû retirer en 2015 de ses rayons en Allemagne un savon liquide baptisé « Ombia, Mille et une nuits", sur lequel figurait une mosquée avec ses minarets; les clients musulmans se plaignaient parce que l'image de leur lieu de culte était associée à un produit destiné aux salles de bains et aux toilettes. «Les symboles sont à manier avec précaution ", concluent Vincent de Féligonde et Marie Alix Maes qui signent cet article, paru le 11 septembre $2017^{4}$. En somme, des consommateurs peuvent protester aussi bien quand un symbole religieux est retiré d'un emballage que quand il y apparait $t^{5}$. Ce qui montre l'importance de l'imagerie publicitaire et son impact sur les enjeux identitaires liant le champ économique non seulement au territoire mais aussi au champ religieux.

En Grèce, la feta est appelée « l'or blanc » : elle constitue un des produits nationaux qui s'exporte le mieux, surtout après l'assignation de l'AOP qui accorde à la Grèce une sorte de monopole commercial au niveau européen. Mais quelle iconographie une marque grecque adopte-elle pour la feta qu'elle exporte? L'emballage de la feta Orino Voskotopi est lui aussi divisé en deux parties. La partie supérieure est marquée par une ligne d'horizon qui fait se rencontrer le ciel et la mer, avec des îles dans le fond. Le logo de la marque (représentant le sommet d'une montagne enneigée) y est inséré à droite ; à gauche, le mot «feta » est écrit en grec en bas, et en caractères latins en haut avec une police de caractères qui renvoie à la Grèce antique ; il est aussi signalé, en grec et en anglais, que ce produit est AOP. La partie inférieure comprend une église à droite, puis un support (qui pourrait être aussi bien une table qu'une assiette) sur lequel sont 
posés un gros morceau et deux tranches de feta; on y trouve une branche de basilic placée sur une des tranches ainsi que trois morceaux de tomate. Les tomates apportent une touche de rouge dans l'ensemble qui est défini par des nuances de blanc, de gris et de bleu (le blanc éclatant de la feta dialoguant avec le blanc-gris des murs de l'église, et la voûte bleue de l'église avec la mer). Le sigle AOP est placé à droite, en bas de l'image.

Photo 5. Publicité sur Internet pour la feta Orino Voskotopi destinée à l'exportation

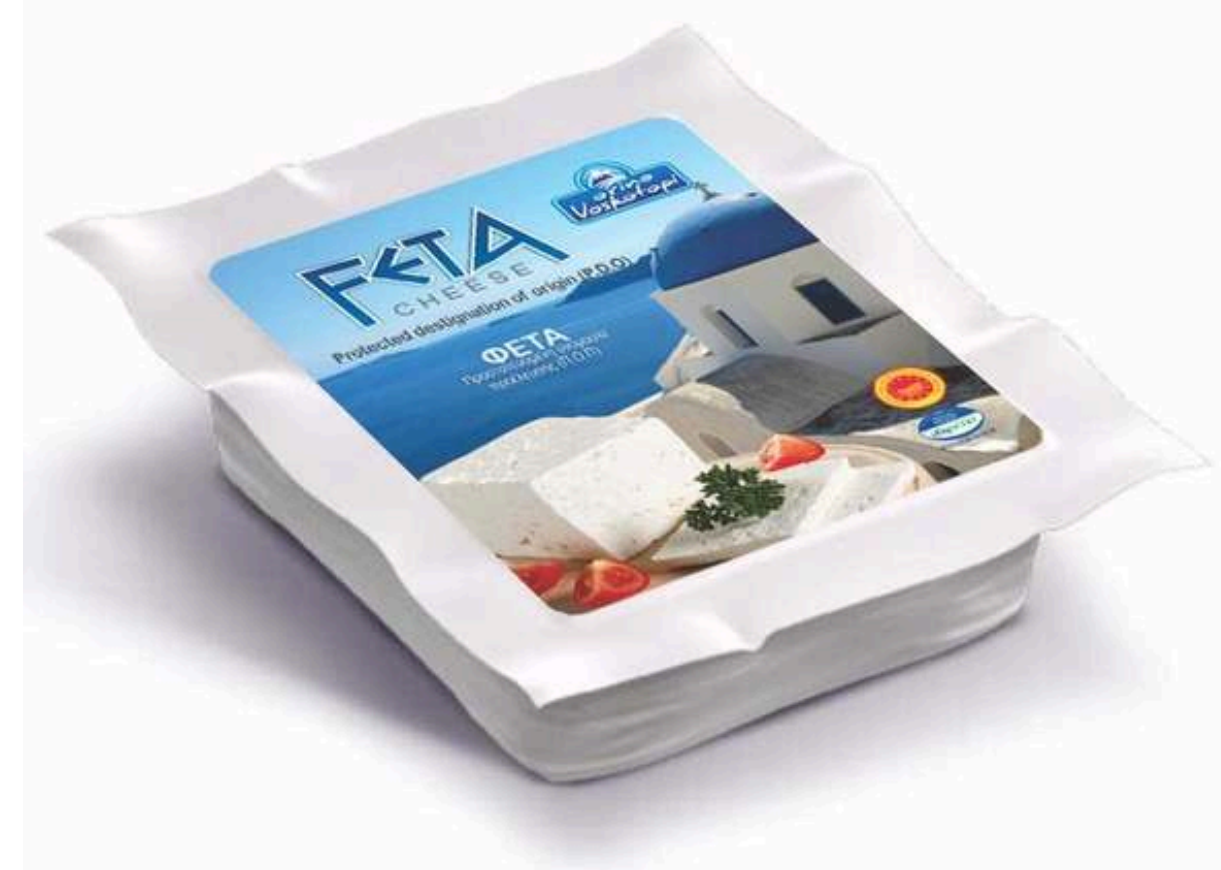

Peu de chose sépare l'imagerie de Sirtakis de celle d'Orino Voskotopi. Même le lieu de fabrication de cette feta (en l'occurrence Lesbos) n'est pas précisé sur ce côté de l'emballage, ce qui montre le peu d'intérêt que cette information représente pour les clients européens auxquels ce fromage est destiné. Nous pouvons toutefois relever trois différences. La première concerne la présence d'une croix sur les églises qui est systématique dans toutes les marques grecques, alors que les marques européennes optent souvent pour sa disparition. Deuxième différence, la palette chromatique de l'image : le paysage cycladique qu'Orino Voskotopi propose est plus réaliste, dans la mesure où une partie de l'église est grise; cette utilisation du gris (qui suggère la dégradation partielle de l'édifice qui n'a pas été repeint récemment) est esthétiquement fonctionnelle, puisqu'elle crée un effet de contraste qui souligne le blanc éclatant de la feta. La question de ce qu'on pourrait appeler «réalisme analogique " se pose également dans le cas de la controverse autour des croix sur les voûtes des églises cycladiques: si les croix ont toujours été là, comment la publicité peut-elle les effacer sur les emballages et ainsi déformer la réalité ? Cette exigence d'une " reproduction fidèle ", voire " photographique ", des paysages est intéressante, étant donné qu'elle s'adresse à la publicité, qui, d'une part, découpe la réalité pour créer des images synecdochiques, et, d'autre part, s'appuie sur des retouches pour rendre plus attrayant le réel. 
La troisième différence est liée aux pratiques de consommation : la feta en cubes (prête à être mélangée dans la salade ou posée sur la pizza) correspond à un mode de consommation européen, alors que les Grecs la coupent en tranches pour accompagner leurs plats de légumes à la sauce tomate ou la salade grecque. Ainsi, Sirtakis montre sur son emballage des cubes, alors qu'Orino Voskotopi présente la feta coupée en tranches. Cela dit, d'autres marques grecques qui exportent adoptent la représentation commune d'une feta coupée en dés, puisque les Européens se sont habitués à consommer la feta sous cette forme, en apéritif ou comme snack.

Photo 6. Publicité sur Internet

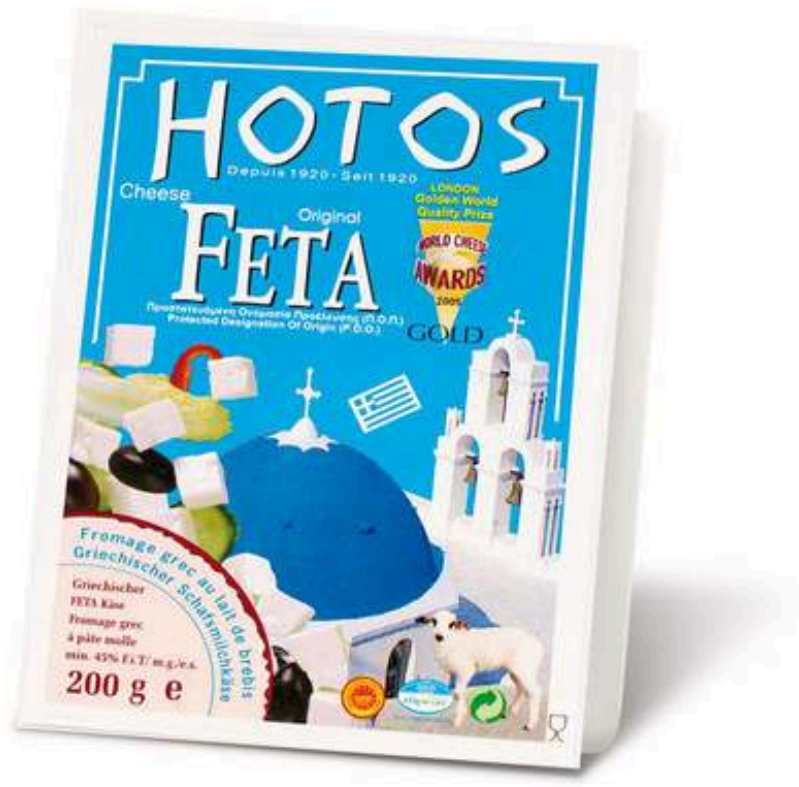

Hotos est une entreprise grecque qui exporte de la feta : les informations sur l'emballage sont en grec, en anglais, en français et en allemand. La croix sur la voûte de l'église et le clocher redoublent les références religieuses, alors que des cubes de feta « volent » dans le paysage.

Il faut savoir qu'avant l'obtention de l'AOP, la Grèce produisait environ 60000 tonnes par an, alors que l'Allemagne et le Danemark en fabriquaient chacun 80000 tonnes. D'ailleurs, un des arguments contre l'assignation de l'AOP était l'incapacité de la Grèce, en tant qu'unique producteur, à satisfaire la demande au niveau européen mais aussi mondial. C'est cette incapacité réelle qui laisse de la place sur le marché pour des marques telles que Salakis qui se positionnent comme des alternatives à la feta AOP. Ces marques de fabrique utilisent des iconographies, des logos, des couleurs et des noms qui renvoient à la Grèce, afin de rendre "grec » un produit qui n'est pas fabriqué en Grèce: plus une identité est approximative, plus elle est suggérée par différents moyens. En effaçant la ligne de démarcation entre ordre visuel et expérience gustative, le packaging présente Salakis comme un substitut de la feta AOP : mêmes paysages sur les emballages, et donc même goût en bouche. 
Le caractère générique du paysage cycladique révèle un manque d'inventivité et un appauvrissement de l'imaginaire; dans le même temps, il permet une économie des moyens utilisés pour définir ce qui renvoie à la Grèce. Or, cette imagerie n'a rien à voir avec le terroir de la feta. D'ailleurs, les marques européennes ne mentionnent pas le lieu de fabrication en Grèce, comme si la question du terroir importait peu. Rappelons que la feta AOP est un produit essentiellement "continental ", étant donné que seule l'île de Lesbos peut aussi en produire. Mais cette production est très petite, de l'ordre de 400 tonnes, quand la Grèce produit environ 120000 tonnes de feta par an, dont 40000 tonnes sont exportées, ce qui rapporte un bénéfice de l'ordre de 200 millions d'euros ${ }^{6}$. Pourtant, même la feta AOP produite à Lesbos, quand elle est exportée (comme dans le cas d'Orino Voskotopi), reproduit cette imagerie cycladique devenue un topos, au lieu de faire figurer sur l'emballage l'architecture locale qui est bien différente. Orino Voskotopi commercialise aussi une feta de Lesbos destinée à la consommation domestique. Sur l'emballage de celle-ci, figure une photographie du port de la ville de Lesbos, avec les dômes caractéristiques de l'église de Saint-Thérapon (construite en 1935, de style byzantin mais avec des éléments architecturaux néoclassiques et gothiques). Ce paysage est très différent de celui que les consommateurs européens ont pris l'habitude de trouver sur le packaging des produits associés à la Grèce.

39 Le paysage cycladique constitue une image de communication uniformisée qui contredit les conditions de fabrication imposées par la dénomination AOP. En somme, il n'y a aucune corrélation entre la réalité économique et l'image publicitaire qui s'avère ici non seulement dépaysante, mais aussi déterritorialisante.

40 Contrairement à ce qui se passe avec la feta "touristique " qui est soumise à un processus de dérégionalisation, l'imagerie de la feta domestique met l'accent sur le terroir. Même si l'attribution de l'appellation AOP définit une zone de production très étendue (l'ensemble de la Grèce continentale), celle-ci a été justifiée par la présence de

Projets de paysage, $19 \mid 2018$ 
conditions géographiques et climatiques particulières : une proportion importante de terres montagneuses, un climat chaud et sec, et des pâturages avec une flore unique (O'Connor et Kireeva, 2003, p.115). Nous quittons donc la mer bleue de la feta « touristique » pour aller vers les montagnes que la feta domestique fait émerger.

\section{Paysages pastoraux et feta domestique}

41 S'il n'y a que des marques de commerce sur le marché européen de la feta, sur le marché domestique nous trouvons aussi des marques de fabrique. Orino Voskotopi est une marque de commerce, produite par l'entreprise Viotyr, qui ne commercialise pas seulement la feta de Lesbos, mais aussi celles d'Épire, de Kalavryta (Péloponnèse), de Karditsa (Thessalie) et de Polykastro (Macédoine). Il suffit de regarder l'étendue de cette offre pour voir émerger l'importance du terroir pour les consommateurs grecs : si dans le cas de la feta de Lesbos ou d'Épire, c'est la provenance régionale qui importe, il est courant de lier la feta non à une région mais à une ville (comme ici avec Kalavryta, Karditsa et Polykastro), la ville représentant toute une tradition pastorale locale.

Quand les marques nationales qui se partagent le marché intérieur mettent sur leur packaging des paysages, ceux-ci sont, dans leur grande majorité, pastoraux : paysage montagnard (avec ou sans habitations), prairie vallonnée, présence du berger et/ou des animaux. De ces paysages, peu sont identifiables. Seuls les ponts épirotes, comme celui qui figure sur la feta d'Épire qu'Orino Voskotopi commercialise, correspondent à une image reconnaissable pour le public grec.

Quand les animaux sont représentés, ils le sont de deux manières : soit statique (en train de brouter ou allongés), soit dynamique (en mouvement, traversant un pont, une plaine ou une rivière, comme dans le cas de la marque Tempi ${ }^{7}$ ). Le fait de montrer les animaux en train de brouter renvoie à la spécificité de la flore de chaque région reconnue comme élément crucial pendant la procédure qui a conduit à l'obtention de l'AOP, puisque les arômes de la flore passent dans le lait, puis dans la feta.

La marque de fabrique Papathanasiou a choisi pour ses emballages une iconographie panoramique: vues d'en haut, une quarantaine de brebis broutent au milieu d'une prairie verdoyante. Sur le site de l'entreprise, qui a été fondée en Grèce centrale en 1987, référence est faite à la richesse des herbes aromatiques locales qui contiennent des Omega-3, mais aussi au mode traditionnel d'élevage ${ }^{8}$ dans cette région. Le visiteur du site apprend ainsi que les chèvres sont principalement élevées dans des régions rocheuses et montagnardes (là où se trouvent les pâturages d'été), alors que les brebis dans des régions relativement plates (correspondant aux pâturages d'hiver). L'iconographie de l'emballage devient alors plus claire, étant donné que cette feta est fabriquée avec $70 \%$ de lait de brebis et $30 \%$ de lait de chèvre - d'où le choix de ne représenter que les brebis dans une prairie.

Les marques grecques, qui montrent les animaux en train de brouter, ont deux options : les insérer dans un paysage ou proposer des "portraits" d'animaux. Sur le pot de saumure de la marque de fabrique Farmakis, on voit à gauche une brebis qui broute et à droite, de profil, une chèvre avec des cornes ; placée sur un tas de pierres, la chèvre est surélevée par rapport à la brebis, qui se trouve sur une surface plane. Les animaux et le paysage sont ici stylisés, un tas de pierres suffisant pour évoquer la montagne. Sur le site Internet de cette entreprise qui a été fondée en Macédoine en 1975, il est précisé 
que la Grèce, au vu de son étendue, est le pays européen qui est caractérisé par la plus grande variété de plantes locales (gigeni fyta) ${ }^{9}$.

La transmission de la tradition et d'un savoir-faire qui se veut «ancestral » inspire aussi l'imagerie de la feta domestique: sur les emballages, le berger est parfois accompagné d'un enfant, ou les brebis de leurs agneaux. Une marque de Lesbos, Lesvigal, met en scène une brebis allongée sur une prairie, sur le dos de laquelle un agneau est monté ; la brebis regarde ailleurs, alors que l'agneau observe le spectateur ${ }^{10}$. En bas de l'image, la feta est servie sur un plateau, accompagnée d'une tomate, de rondelles d'oignons et de deux tranches de concombre. Cette iconographie rappelle celle de la feta "touristique ", à la différence que le plateau qui devient un balcon ne donne pas sur la mer et l'architecture cycladique mais sur une scène pastorale. Deux autres entreprises jouent avec cette idée du balcon: Papathanassiou avec son panorama pastoral, et Roussas avec la marque Terra Aeolica destinée à l'exportation, où l'on voit le ciel bleu, une prairie avec un arbre au fond et trois brebis qui broutent autour de l'arbre. Dans tous ces cas, l'emballage est divisé en deux parties (horizontales pour Lesvical et Terra Aeolica, verticales pour Papathanassiou qui met le plateau de la feta non pas en bas de l'image mais à droite). Cette forme d'organisation visuelle est marquée par la coexistence d'un arrière-plan (le paysage) et d'un gros plan (le plateau avec la feta qui joue le rôle du balcon).

47 L'entreprise Chelmos, qui a été créée en 1979 et qui est la plus importante industrie laitière du Péloponnèse, exporte aussi sa feta à l'étranger : ici, la partie latérale de l'emballage est en allemand. Nous retrouvons l'idée du balcon qui donne cette fois sur une prairie à perte de vue. Cette représentation (avec ciel bleu et ligne d'horizon) établit un parallèle entre mer et prairie, que l'absence d'arbres ou d'animaux ne fait que renforcer. S'agirait-il d'un compromis visant à remplacer le paysage cycladique par une imagerie pastorale plus conforme aux «attentes visuelles» des consommateurs européens? 


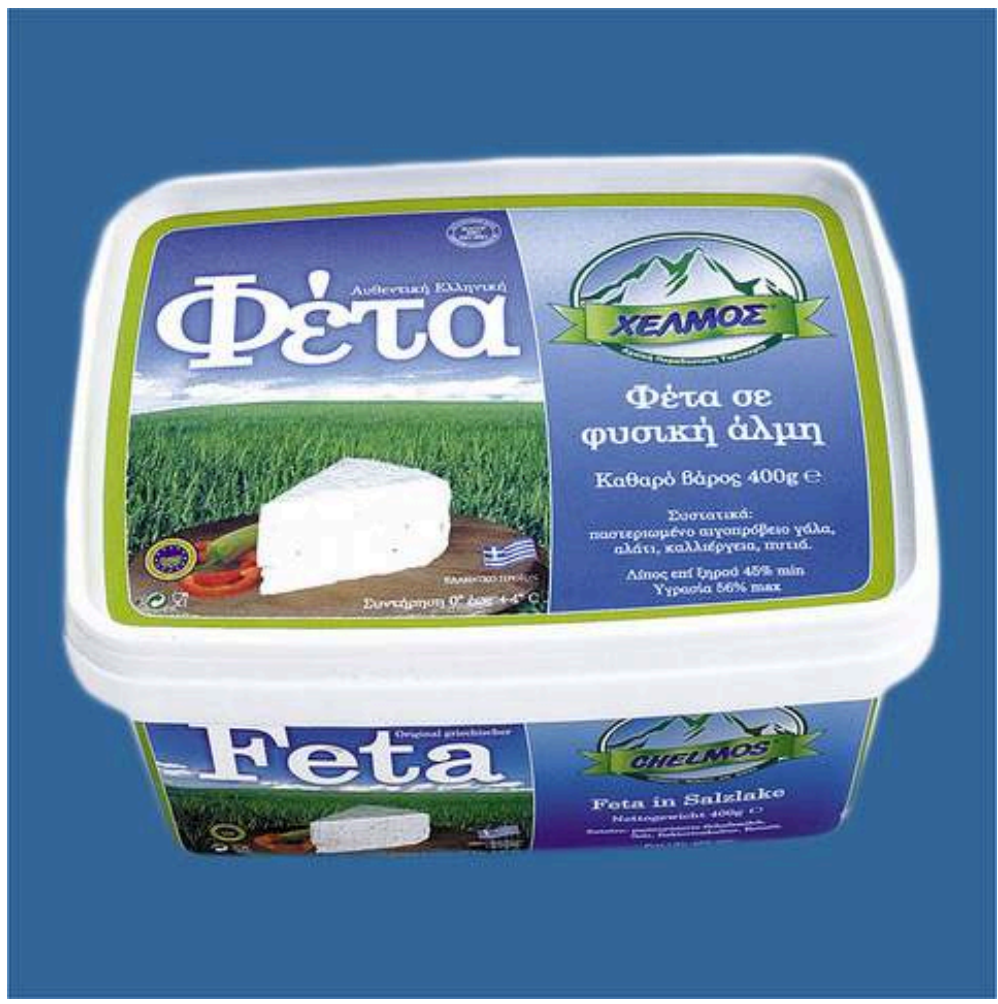

La coupe triangulaire de la feta, qui rappelle celle d'un fromage rond comme le camembert, est tout à fait inhabituelle. structure de ceux qui se vendent depuis les années 1990 en Europe. L'imagerie de la feta «touristique » semble donc faire des émules même sur le marché grec, sans pour autant remplacer le paysage pastoral par des références cycladiques. Cela dit, le fait de mettre au premier plan la représentation du produit (ou d'un de ses ingrédients) et au second plan un paysage jugé évocateur constitue une pratique courante dans le packaging ${ }^{11}$.

Depuis quelques années, le répertoire de la feta est devenu extensible. La question que pose la partie suivante est de savoir dans quelle mesure une situation relative de monopole, comme celle créée par l'obtention de l'AOP, pousse à une plus grande production de variantes qui permettent aux marques de se différencier.

\section{Diversification de l'offre et variantes iconographiques}

De nouvelles gammes, comme la feta affinée, ont fait leur apparition sur le marché tant domestique qu'européen. Les conditions de maturation sont parfois suggérées de manière explicite : le packaging imite alors le bois du tonneau (vareli) dans lequel la feta doit rester pendant une période minimum de trois mois. La spécificité de cette feta affinée, dont le goût est plus prononcé et la pâte plus dure, est accentuée par l'adjectif qui lui est systématiquement attribué : feta varelissia pour les Grecs, et barrel aged pour les Européens. 
La marque Karalis a choisi de faire figurer sur son emballage un tonneau de bois derrière les tranches de feta qui sont posées horizontalement les unes sur les autres: du pain, des légumes, de l'ail, du persil, des olives et du raisin vert entourent les tranches - le raisin correspondant à une pratique de consommation typiquement grecque. En bas à droite, on voit même un pont épirote, derrière lequel un troupeau broute.

Photo 8. Feta varelissia Karalis dans un supermarché athénien (Nea Kifissia)

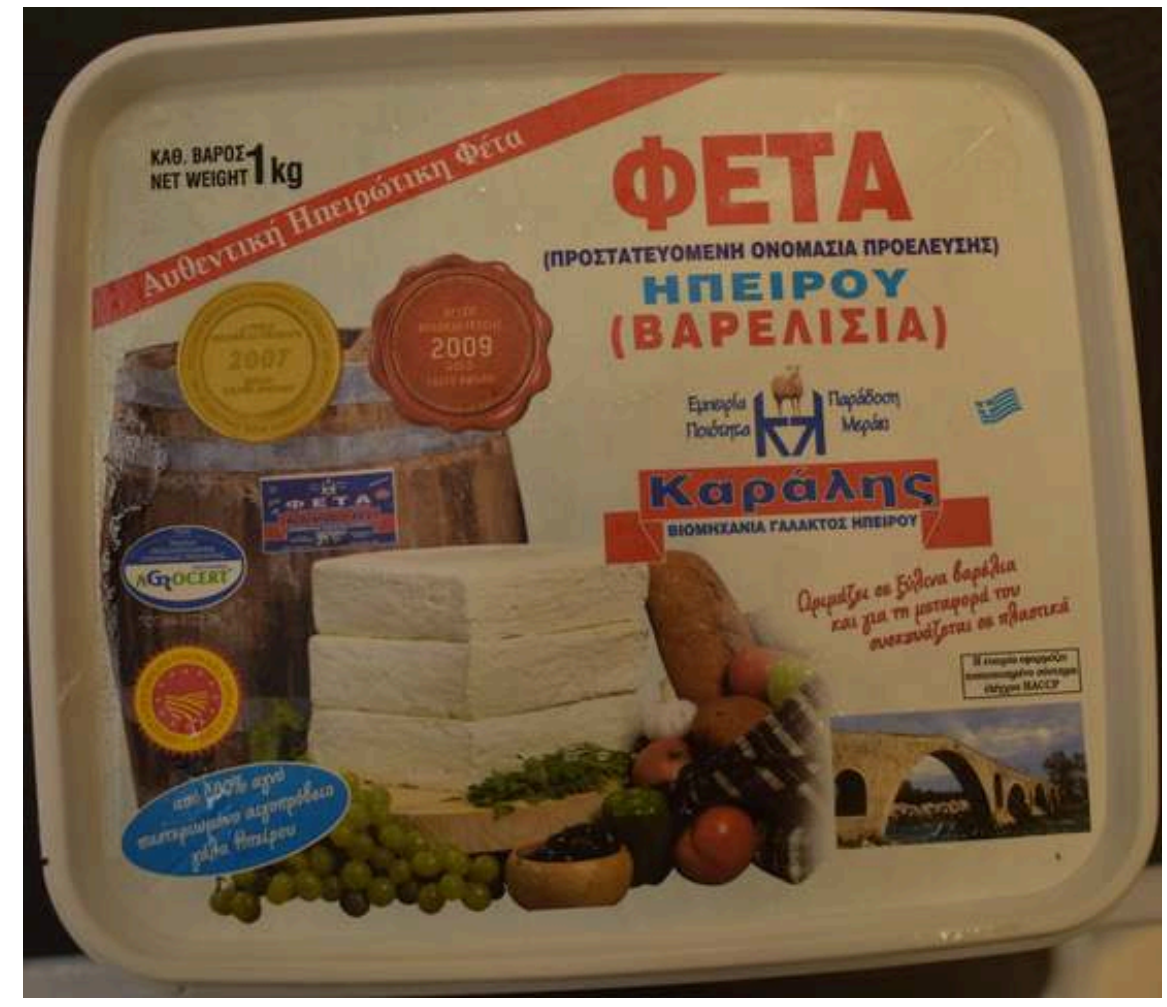

25 septembre 2018 .

Photographie d'Alkisti Kyriakopoulou.

52 L'entreprise Karalis, qui a été fondée en 1947 en Épire, est la plus grande au niveau national en ce qui concerne la production et l'exportation du fromage kefalograviera AOP (assignée en 1996). Selon Kostas Karalis, les exportations vers les États-Unis et le Canada commencent en 1982; cette initiative le pousse alors à investir dans la production de la feta, puisqu'elle est très demandée par les immigrés grecs installés aux Amériques (Greek foods success stories, 2015, p. 124-125). Dans ce cas, la demande pour une feta " touristique » semble être à l'origine de la production de ce fromage par une entreprise grecque spécialisée dans la fabrication du kefalograviera.

Quant à la feta affinée d'Épire qu'Orino Voskotopi commercialise, elle met en scène sur l'emballage un berger et, à ses côtés, un enfant - tous deux habillés de manière traditionnelle. Ils sont au sommet d'une montagne, aucun animal n'est visible aux alentours, ce qui accentue encore le sentiment d'isolement. Le choix de cette iconographie pour une feta qui a au moins cinq mois de maturation, comme cela est précisé sur l'emballage (avec le chiffre 5 en blanc dans un cercle bleu qui le fait ressortir encore plus), est significatif : c'est comme si les bergers, appuyés sur leurs houlettes, attendaient patiemment la maturation du fromage. Cette imagerie semble 
suggérer deux choses : d'un côté, la patience dont il faut faire preuve pour avoir une feta affinée ; et de l'autre, la transmission d'une tradition pastorale séculaire de père en fils. Le paysage représente ici le lent écoulement du temps qui n'a rien à voir avec le rythme effréné des villes et l'impatience de ceux qui y habitent.

Certaines entreprises grecques exportent également ces produits. Par exemple, Roussas, une entreprise épirote fondée en 1952, exporte de la feta barrel aged, mais aussi deux autres variantes, l'organic feta et la feta cubes. Roussas, qui exporte $90 \%$ de sa production, est une des rares entreprises grecques qui n'utilise pas l'imagerie cycladique : les emballages de ses produits sont définis soit par une imagerie pastorale (comme avec sa marque Terra Aeolica que nous avons examinée plus haut), soit par une imagerie monastique, avec sa marque Mt Athos.

Photo 9. Rayon des fromages dans une autre épicerie turque de Bruxelles (quartier d'Ixelles)

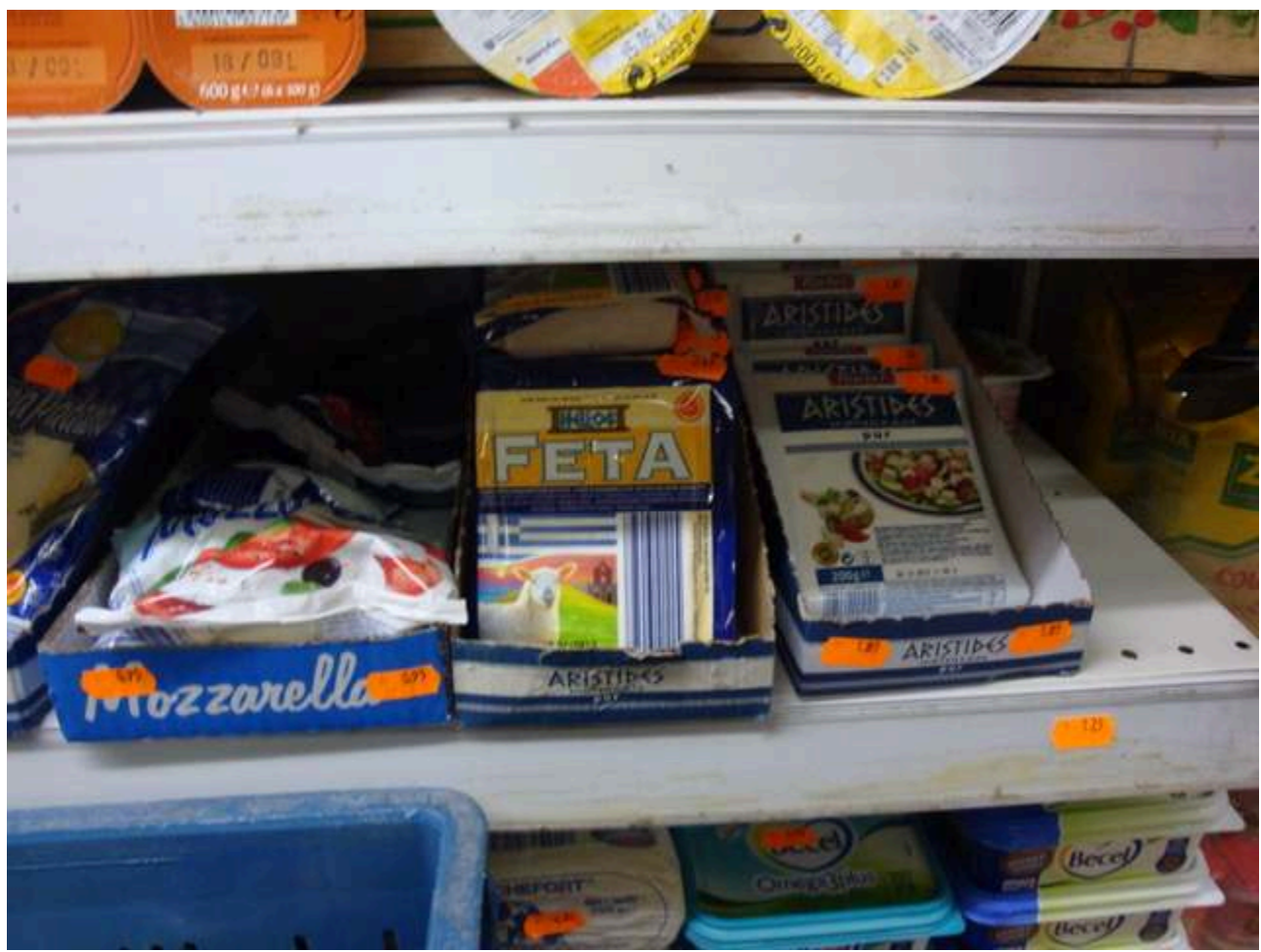

L'emballage au milieu montre comment il est possible de combiner imagerie cycladique (clocher, mer, coucher de soleil) et imagerie pastorale (colline verdoyante et brebis qui regarde le consommateur). Notons également la proximité de la feta et de la mozzarella dans le rayon (dont les emballages, quand ils sont neutres, se rassemblent beaucoup), 5 septembre 2012.

Photographie de Katerina Seraïdari.

Sur le marché européen, les produits de ce type se multiplient. Même la marque allemande Gazi, qui propose une gamme de produits turcs comme nous l'avons vu dans la première partie, vend une feta AOP barrel aged, choisissant pour son emballage un paysage méditerranéen panoramique avec une haie de cyprès et un village au premier plan, ainsi qu'une colline baignée par le soleil au second plan.

Ces produits représentent des nouveautés à valeur ajoutée : la feta est déjà découpée en cubes et d'autres ingrédients y sont ajoutés, comme de l'huile, des herbes, des tomates, des olives, des poivrons ou du basilic. La feta AOP est toujours fabriquée en Grèce, mais les ingrédients rajoutés n'ont plus rien à voir avec la Grèce. Le statut de la marque 
change également, dans la mesure où le produit final constitue un mélange de différents ingrédients: de simple marque de commerce, elle devient marque de fabrique. La production de ces variantes transforme inévitablement la situation relative de monopole que crée l'AOP : la feta devient ainsi une matière première qui peut être déclinée.

Cette évolution, qui fait monter les prix des produits proposés, modifie également l'aspect iconographique des emballages. La marque allemande Greco commercialise une feta en cubes avec des tomates et une autre avec des herbes. La composition de l'emballage des deux produits est similaire. Elle est divisée verticalement en deux parties : à gauche, on voit une église cycladique (avec croix), ainsi que le sigle AOP en bas; et à droite, trois grosses tomates (ou des herbes, pour le second produit) qui sont posées sur un tas de cubes de feta ; au milieu de l'image, une brebis dans un médaillon, qui semble marcher sur les cubes de feta, regarde le consommateur. Ce qui distingue les deux emballages est leur couleur : tout est rouge-rose-blanc dans le premier cas, et vert-blanc dans le second. La voûte bleue de l'église cycladique est donc devenue rose ou verte, selon l'ingrédient ajouté à la feta.

\section{Photo 10. Publicité sur Internet pour la marque Greco}

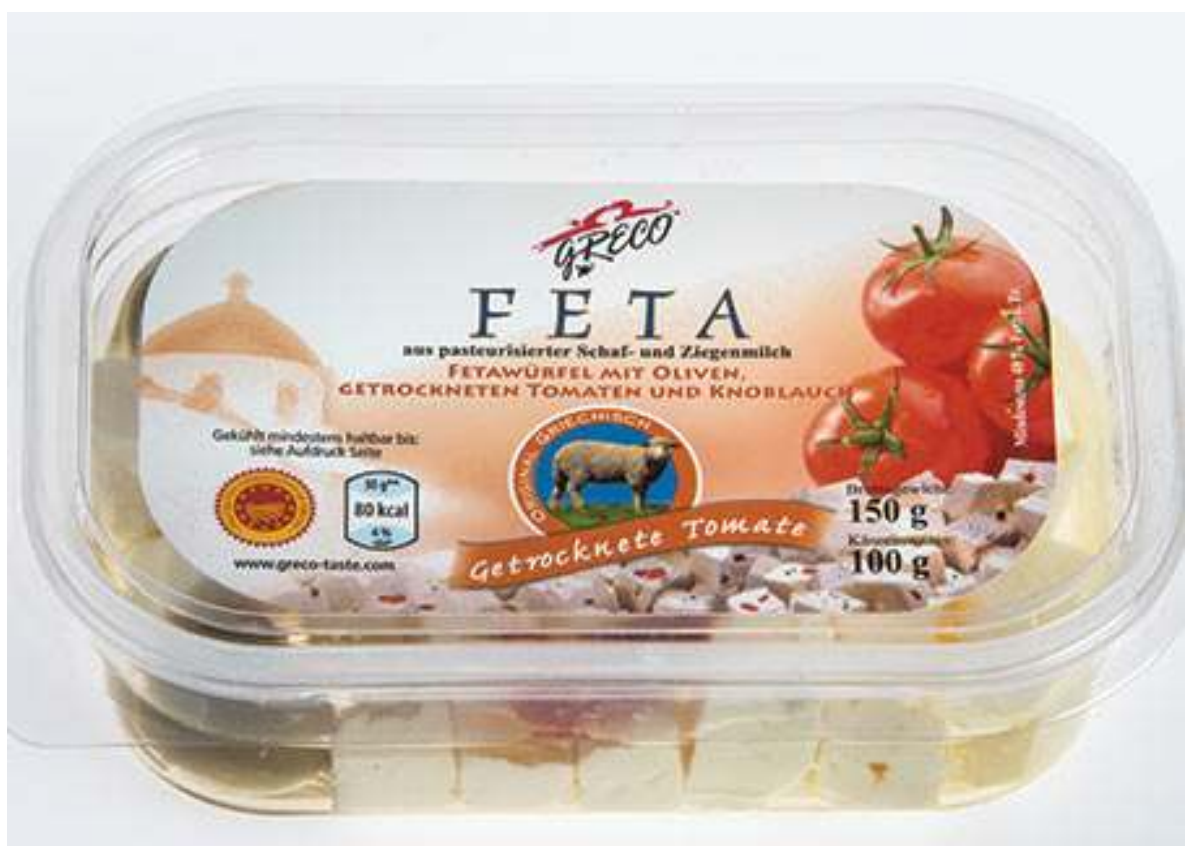

Si l'emballage est le signe de reconnaissance d'une marque, tous les emballages se ressemblent dans le cas de la feta "touristique». Les deux variantes de la marque Greco, d'une part, montrent comment le changement de la palette chromatique sur les emballages résout ce problème d'uniformisation et d'image générique ; et, d'autre part, proposent une nouvelle manière d'utiliser le paysage, dans la mesure où l'exigence d'un «réalisme analogique » est ici clairement estompée : une fois devenue rose ou vert, le paysage évoque, plus qu'il représente. Alors que le signe de qualité AOP et l'emballage "cycladique» induisent le principe de similarité, les nouvelles gammes et leur packaging poussent vers la singularisation. 


\section{Deux espace-temps : de la pâture à la plage} de l'hiver et le printemps. C'est probablement pour cette raison que sur les montagnes représentées ne reste que peu de neige. Sur l'emballage de la feta de Minerva-Chorio ${ }^{12}$, on distingue un bouquetin sur un fond de montagnes dont les sommets sont encore enneigés, alors que l'herbe couvre déjà un plateau plus bas. 
Photo 11. Feta Chorio dans un supermarché athénien (Nea Kifissia)

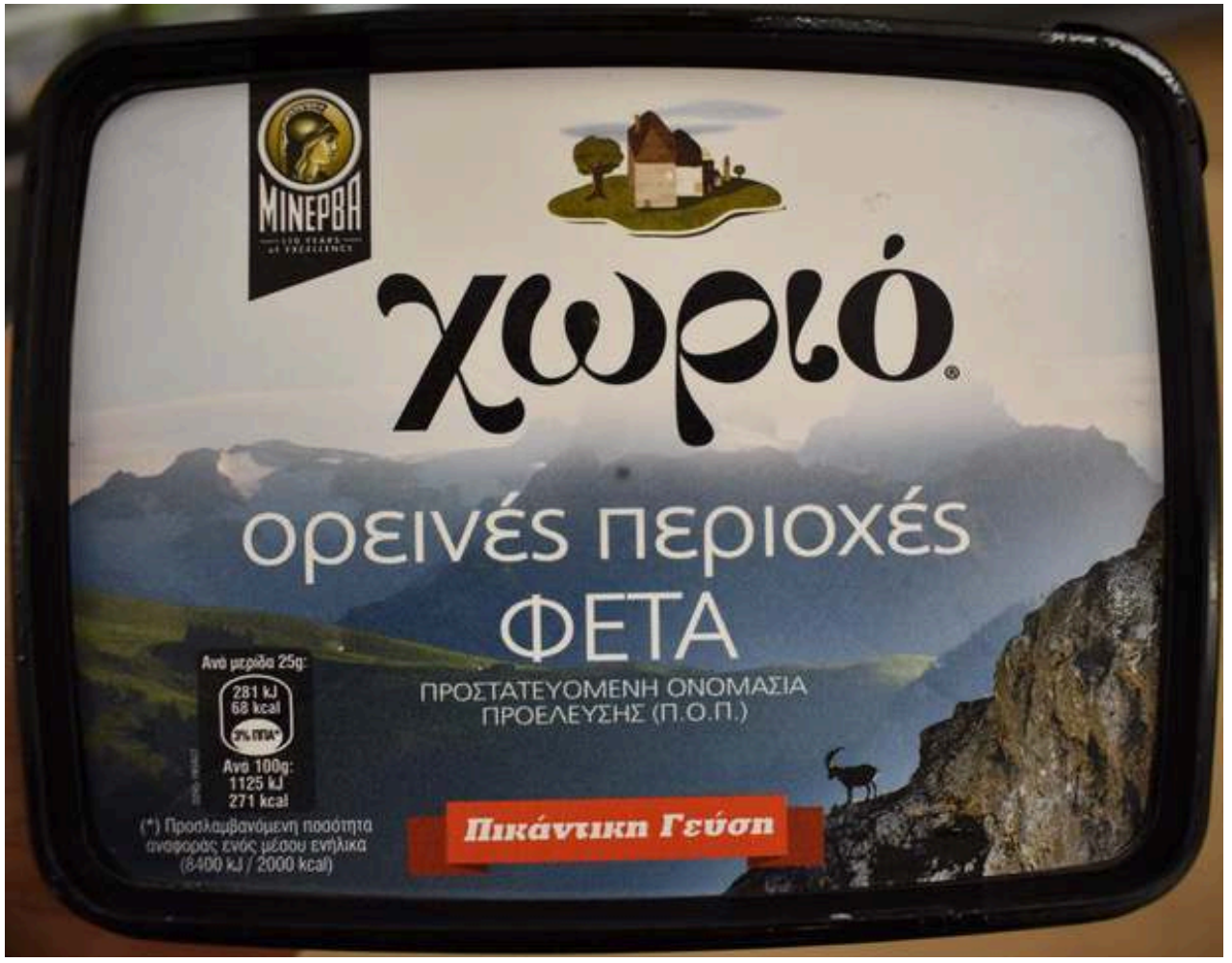

25 septembre 2018.

Photographie d'Alkisti Kyriakopoulou.

63 L'imagerie pastorale de la feta domestique a le goût de la précision, inscrivant le paysage dans le temps et indiquant la bonne saison, le moment propice pour la fabrication d'un bon fromage. De même, la présence des brebis et des chèvres sur les emballages domestiques vise à souligner la tromperie dont les consommateurs étaient victimes avant l'obtention de l'AOP: la plupart des marques européennes, alors importées également en Grèce, fabriquaient une feta avec du lait de vache, qui était naturellement jaunâtre et devait ensuite être blanchie avec des substances colorantes (Petridou, 2005).

emballages de la feta domestique sont plus informatifs et descriptifs. Toutefois, le message que leur imagerie véhicule est également trompeur, dans la mesure où ces images bucoliques occultent les processus de modernisation et de mécanisation. Ces emballages ont peut-être le goût de la précision en ce qui concerne la saison de la production du lait, mais sûrement pas pour ce qui est du temps dans son ensemble, puisqu'il y paraît figé et immémorial. Bien sûr, dans le cas de certaines marques, les troupeaux sont encore élevés en montagne, mais les bergers ne sont pas habillés de manière traditionnelle.

Le paysage publicitaire de la feta construit chaque fois un certain rapport au temps, de la consommation et/ou de la production, l'acheteur étant transformé en vacancier ou en témoin de l'élevage en montagne. Ce qui fait la spécificité de la feta domestique est son inscription dans un registre mémoriel et nostalgique : elle est censée transporter le consommateur non seulement dans un espace autre, mais aussi dans un temps autre, celui d'un passé révolu (« manger de la feta, c'est retrouver le bon vieux temps »). Par 
sa promesse de faire resurgir le passé, le produit est censé pouvoir changer la perception du temps qui ne serait plus irréversible.

Alors que les emballages de la feta «touristique » insistent sur la capacité de ce fromage à dépayser, ceux de la feta domestique évoquent un monde rural qui serait resté intact. Qu'il s'agisse de l'évasion vers une île ou d'un retour aux racines, les emballages imposent une fiction dans les deux cas, en représentant des lieux de liberté et de pureté, coupés du monde et loin de tout. Mais une différence importante subsiste : le paysage cycladique constitue un décor, tandis que les scènes pastorales renvoient à des activités économiques qui façonnent réellement le paysage.

\section{BIBLIOGRAPHIE}

Barrey, S., Cochoy, F. et Dubuisson-Quellier, S., « Designer, packager et merchandiser : trois professionnels pour une même scène marchande ", Sociologie du travail, vol. 42, 2000, p. 457-482.

Barthes, R., « Rhétorique de l'image », Communications, n 4, 1964, p. 40-51.

Bigando, E., «La synecdoque paysagère, une notion pour comprendre les représentations des paysages viticoles bourguignon et bordelais ", Sud-Ouest européen, t. 21, 2006, p. 83-93.

Cochoy, F., Une sociologie du packaging ou l'âne de Buridan face au marché, Paris, Presses universitaires de France, 2002.

Greek food success stories, vol. A, Halandri-Athènes, Koufalis media Corporation, 2015.

Dobremez, L., Rapey, H., Candau, J. et Ginelli, L., « Paysages et produits certifiés : un lien évident ? Pratiques et discours d'éleveurs en Auvergne et Morvan », Fourrages, n² 216, 2013, p. 293-304.

Frayssignes, J., «L'AOC Roquefort : une filière emblématique », dans Hervieu, B. et le Centre international de hautes études agronomiques méditerranéennes, MediTERRA 2007. Identités et qualité des produits alimentaires méditerranéens, Paris, Presses de Sciences Po, coll. « Annuels », 2007, p. 147-184.

Menadier, L., «Que révèlent "parcelles préférées” et “coins de paradis" sur les caractères d'un produit? Méthode d'analyse du point de vue paysager d'agriculteurs en zones AOC fromagères de moyenne montagne ", Projets de paysage, juillet 2010, n 4, URL : http://www.projetsdepaysage.fr/ fr/que_revelent_parcelles_preferees_et_coins_de_paradis_sur_les_caracteres_d_un_produit_.

Michelin, Y., «L'approche sémiologique au service de la mise en évidence du lien produit agricole-paysage », Actes sémiotiques, 2008, URL : http://epublications.unilim.fr/revues/as/3409.

Moser, R., « Les étiquettes anciennes du vin d'Alsace », Revue d'Alsace, n 137, 2011, p. 109-134.

O' Connor, B., The law of geographical indications, London, Cameron May International Law Policy, 2004

O' Connor, B. et Kireeva, I., "What's in a name ? The "feta" cheese saga ", International Trade Law and Regulation, vol. 9, $\mathrm{n}^{\circ}$ 4, 2003, p. 110-121. 
Petridou, E., « Au pays de la feta. Négociation de la grécité dans le contexte européen », Ethnologie français, vol. 35, 2005/2, p. 255-265.

Poulain, J.-P., Sociologies de l'alimentation. Les mangeurs et l'espace social alimentaire, Paris, Presses universitaires de France, 2002.

Seraïdari, K., La Ville, la Nation et l'Immigré. Rapports entre Grecs et Turcs à Bruxelles, Paris, L'Harmattan, 2012.

Tapia, S. de, « Les circuits d'approvisionnement des commerces turcs en Europe. Formation d'un champ transnational ", dans Mullet, L. et Tapia, S. de (dir.), La Création d'entreprise par les immigrés. Un dynamisme venu d'ailleurs, Paris, L'Harmattan, 2005, p. 53-89.

Tsiboukas, K. et Vallerand, F., « Formes de filières lait-fromage de petits ruminants en Grèce et leurs perspectives de développement », dans Dubeuf, J.-P. (dir.), L'Évolution des systèmes de production ovine et caprine : avenir des systèmes extensifs face aux changements de la société, Zaragoza, CIHEAM, 2004, p. 307-315.

\section{NOTES}

1. Les opérateurs industriels qui conditionnent la feta en Grèce peuvent aller jusqu'à tripler le prix net du produit en le vendant en barquettes ; leurs profits sont ainsi maximisés par rapport à la vente à la coupe (Tsiboukas et Vallerand, 2004, p. 310).

2. URL : http://nevamilk.com/en/catalog/sirtaki/. Consulté le 30 janvier 2018.

3. Qui montre un berger âgé, cheveux blancs, moustache blanche, qui tient une houlette - image indicielle suggérant une tradition ancestrale.

4. URL : https://www.la-croix.com/Economie/Entreprises/Eglises-sans-croix-produits-grecqueLidl-nest-pas-seul-cause-2017-09-11-1200875989.

5. La présence d'une mosquée sur un savon semble être moins tolérée que celle sur une feta. En effet, la marque Gazi utilise cette iconographie sur le packaging de ses fromages, mais celle-ci n'a jamais été mise en cause par mes informateurs turcs de Bruxelles. Le fait que la marque Gazi soit considérée comme turque joue certainement un rôle dans cette acceptation.

6. Selon un article publié sur le site Internet «Greek gastronomy guide » le 13 juin 2017, URL : http://www.greekgastronomyguide.gr/en/greek-feta-cheese-product/.

7. La vallée de Tempi est parcourue par le Pinios, troisième plus grand fleuve de Grèce. La rivière sur l'emballage évoque clairement une particularité de la région: il y a donc bel et bien une correspondance entre le lieu de production et le paysage représenté sur ce packaging.

8. Certaines entreprises grecques se réfèrent à une tradition qui leur serait léguée par des ancêtres valaques ou sarakatsans. Cette thématique, qui met en scène des populations nomades traversant les Balkans et les frontières entre les différents États qui les composent, pourrait servir de contre-argument à l'assignation d'une identité exclusivement grecque à ce fromage.

9. Quand Dobremez, Rapey, Candau et Ginelli (2013, p.296) ont demandé à des éleveurs d'Auvergne de photographier les éléments de paysage qu'ils souhaitaient voir associer à leurs produits, certains d'entre eux ont photographié la flore des prairies. Ce n'est donc pas un hasard si de petits entrepreneurs grecs, souvent directement impliqués dans l'élevage, mettent l'accent sur la diversité de la flore.

10. La présence d'animaux «mignons » ou de paysages idylliques sur les emballages de la feta correspond clairement à une sensibilité citadine.

11. Pour un exemple similaire où il est question des gâteaux diététiques dont l'emballage montre au premier plan le chocolat et au deuxième plan un paysage désertique (évoquant la détente), voir Barrey, Cochoy et Dubuisson-Quellier (2000, p. 463). 
12. Fondée en 1900, Minerva est une entreprise spécialisée dans la production d'huile, qui a décidé en 2008-2009 de se lancer dans le marché des produits laitiers avec la marque Chorio.

\section{RÉSUMÉS}

La juxtaposition des emballages de différentes marques grecques et européennes, qui fabriquent et commercialisent la feta (devenue AOP depuis 2003), permet d'analyser l'imagerie que leur packaging véhicule. Alors que les publicités paysagères de la feta qui est vendue en dehors de la Grèce évoquent l'évasion et le plaisir d'un voyage dans les Cyclades, celles de la feta commercialisée en Grèce insistent sur l'authenticité et le caractère séculaire d'une production pastorale artisanale. Malgré leurs divergences, les paysages représentés renvoient dans les deux cas moins à un espace qu'à une origine - simplement grecque, dans le cas de la feta «touristique»; locale et régionale, dans le cas de la feta domestique qui met l'accent sur l'importance du terroir.

The juxtaposition of the packaging of different Greek and European brands that produce and market feta cheese (which has become a PDO since 2003), makes it possible to analyse the imagery their packaging conveys. While the landscapes in advertisements for feta cheese sold outside Greece evoke the pleasure of a trip to the Cyclades, those landscapes used in advertisements for feta cheese sold in Greece emphasize the authenticity and secular nature of traditional pastoral production. In spite of their differences, the landscapes represented in both cases refer to an origin rather than to a place - a Greek origin in the case of "tourist" feta and a local and regional origin in the case of domestic feta, which emphasizes the importance of the terroir.

\section{INDEX}

Keywords : cheese, advertising, PDO certification, packaging, Greece

Mots-clés : fromage, publicité, certification AOP, emballages, Grèce

\section{AUTEUR}

\section{KATERINA SERAÏDARI}

Katerina Seraïdari est docteure en anthropologie sociale et membre associé du Centre d'anthropologie sociale de Toulouse (LISST). Ses recherches portent sur deux grands thèmes : le culte des icônes en Grèce et leurs fêtes patronales ; les immigrés grecs et turcs à Bruxelles et leurs activités commerciales. k.seraidari[at]gmail[dot]com 